\title{
Structural Bioinformatics: A Brief Overview
}

\author{
Long Pei* \\ Department of Mathematical Sciences, University of Southern California, USA \\ *Corresponding author: Long Pei, Department of Mathematical Sciences, University of Southern California, USA.
}

Submission: 非June 18, 2018; Published: 㭗June 26, 2018

\section{Introduction}

In the past decades, many advanced bio-technologies have accelerated frontier research to explore biological systems including cell, the basic unit of life. Experimental exploration of cellular environment, however, will be difficult as it requires not only huge labor efforts and costs but also large number of samples for reproducible research. With large volume of available biological data generated, bioinformatics becomes an important hybrid science subject to support systematic and comprehensive data storage, processing and analysis. In joint with statistics and algorithms, biological data such as DNA sequences have been more efficiently processed by bioinformatics methods. Unlike sequential data, biological structures are usually more dynamic and stochastic, thus, structural biology is more challenging a task for bioinformatics.

Structural bioinformatics is the branch of bioinformatics which specifically targeting at the analysis or prediction for three-dimensional structure of biological systems. Structural bioinformatics deal with biological systems at different scales, from molecules to cellular environment. Many types of cellular compartments, for example, the organization of the cell cytoplasm and the structural organization of the genome in its nuclear environment could be formalized by integrative structural bioinformatics analysis. Structural bioinformatics usually involves following steps:

A. Collecting of biological data, either high throughput sequencing data or imaging data.

B. Building computational model, can be structural simulation, optimization or alignment.

C. Interpreting the model results from structural biology perspectives.
D. Providing insights for next iteration of experimental design.

Recently developed technologies has enabled high throughput sequencing and imaging data collection. For example, Chromosome conformation capture techniques [1] are used to analyze the spatial organization of chromatin in a cell based on high throughput sequencing data. By quantifying interactions between genome loci, we are able to infer the three-dimensional folding mechanism of the genome. For the imaging data, Cryo-electron tomography [2] is an important tool to study structures of macromolecular complexes in close to native states based on 3D back-projection of a series of the projected 2D images of the structure.

Many statistical learning methods and computational algorithms could be applied towards building structural bioinformatics models. Molecular dynamics, for example, could be useful to simulate the distributions of biological molecules and the interactions among them. Clustering methods, could be applied to detect subtypes of conformations among population data. Convolution neural networks is a good fit for image processing. Graph-based learning methods is a suitable way to perform structural alignments. With statistical learning methods and computational algorithms, structural bioinformatics has the great potential to help save labor, cost and time. Moreover, it could help providing insights for next iteration of experimental designs so as to collect more biological data.

\section{References}

1. Lesne A, Riposo J, Roger P, Cournac A, Mozziconacci J (2014) 3D genome reconstruction from chromosomal contacts. Nat Methods 11(11): 11411143.

2. Lučić V, Rigort A, Baumeister W (2013) Cryo-electron tomography: The challenge of doing structural biology in situ. J Cell Biol 202(3): 407-419. 
Creative Commons Attribution 4.0

International License

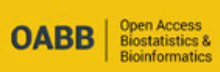

For possible submissions Click Here Submit Article
III

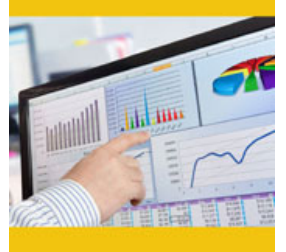

\section{Open Access Biostatistics \& Bioinformatics}

\section{Benefits of Publishing with us}

- High-level peer review and editorial services

- Freely accessible online immediately upon publication

- Authors retain the copyright to their work

- Licensing it under a Creative Commons license

- Visibility through different online platforms 